\title{
Dispersion-controlled optical group delay device by chirped photonic crystal waveguides
}

\author{
Daisuke Mori and Toshihiko Baba ${ }^{\text {a) }}$ \\ Department of Electrical and Computer Engineering, Yokohama National University, 79-5 Tokiwadai, \\ Hodogayaku, Yokohama 240-8501, Japan
}

(Received 13 February 2004; accepted 16 June 2004)

\begin{abstract}
Previously, we proposed and demonstrated chirped photonic crystal waveguides, in which some structural parameters are gradually changed so that the photonic band characteristic is smoothly shifted. In this letter, we discuss an optical delay line composed of two index-chirped waveguides in a directional coupler structure. A large delay is realized by a low average group velocity of $<_{c / 350}$ near the band-edge with almost perfect dispersion compensation even for a short optical pulse. Finite difference time domain simulation demonstrates that such a device is possible in a practical design. (C) 2004 American Institute of Physics. [DOI: 10.1063/1.1783014]
\end{abstract}

The photonic crystal (PC) line defect waveguide ${ }^{1-4}$ allows ultrasmall components 5,6 and low group velocity $v_{g}$ (Ref. 7) due to its strong optical confinement by the photonic bandgap (PBG). A low $v_{g}$ near the photonic band edge is not only effective for a delay line but for the enhancement of various effects, such as optical amplification/absorption, electro-/magneto-optic effects, and nonlinear effects. However, the low $v_{g}$ cannot simply be applied for short pulses and high-speed modulated signals, since such optical input has a wide spectrum, while the frequency range showing an ultralow $v_{g}$ (e.g., $<c / 100$ ) is very narrow (e.g., $<0.1 \%$ of the center frequency). ${ }^{7}$ Furthermore, the band edge also exhibits very large group velocity dispersion (GVD). In order to maintain the wave form of the pulse, effective compensation of the GVD is indispensable. Previously, we have proposed a chirped PC waveguide, in which some structural parameters are gradually changed along the waveguide, and so the photonic band characteristics are smoothly shifted. ${ }^{8}$ It allows ultralow $v_{g}$ and the corresponding light localization for any frequencies at different positions, as was observed in a finite-difference time-domain (FDTD) calculation and in a preliminary experiment. ${ }^{8}$ In this letter, a compact delay line based on the chirped PC waveguide is proposed. This device achieves a large delay in a wide spectral range with almost perfect compensation of the GVD. For this reason, we call it the group delay device. Very recently, a similar function based on an external active modulation of a PC has been theoretically discussed. ${ }^{9}$ Such active control is not necessary for our device. In the following, we first explain two basic elements (the index chirping and the directional coupler) and the principle of the device. Next, we describe its practical design and theoretical demonstration by the FDTD method. Finally, we discuss the potential and technical issues of this device for a nanosecond-order long delay.

In the proposed device, a two-dimensional (2D) PC of triangular lattice airholes and the chirping of background refractive index are used, as illustrated in Fig. 1(a). We have also investigated other chirp parameters, e.g., airhole diameter $2 r$ and lattice constant $a$. ${ }^{8}$ However, we noticed in the FDTD calculation that the index chirping gives the smoothest shift of the band characteristic. Let us assume a single

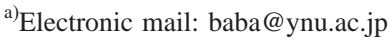

line defect PC waveguide, ${ }^{1}$ as an example. It normally exhibits an upward band profile of guided mode, as shown schematically in Fig. 1(a). The band shifts to the higher frequency side, when the index decreases in the propagation direction. Therefore, guided light gradually slows and finally reaches the band-edge condition. Here, the light is localized, but is soon reflected and moves backward in the waveguide with some delay. By optimum design, this compensates the GVD, as in a chirped fiber grating. Next, let us consider a directional coupler composed of such chirped waveguides, as shown in Fig. 1(b). Two waveguides, A and B, have different structures and characteristics; $\mathrm{A}$ and $\mathrm{B}$ have upward and downward band profiles, respectively, but their band edges are always equal. Similar to the above-mentioned case, the guided light in waveguide A reaches the band-edge condition and is localized. Under this condition, the frequency $\omega$ and the wave number $k$ in the propagation direction agree between the waveguides. Therefore, efficient directional coupling occurs and localized light moves to waveguide B. This time, light is not reflected but rather moves forward in waveguide $\mathrm{B}$ with increasing $v_{g}$ due to the chirping. The delay by localization takes place for any frequencies at different positions. If the upward band profile of $\mathrm{A}$ and the downward band profile of $\mathrm{B}$ are symmetric, the GVD near the band

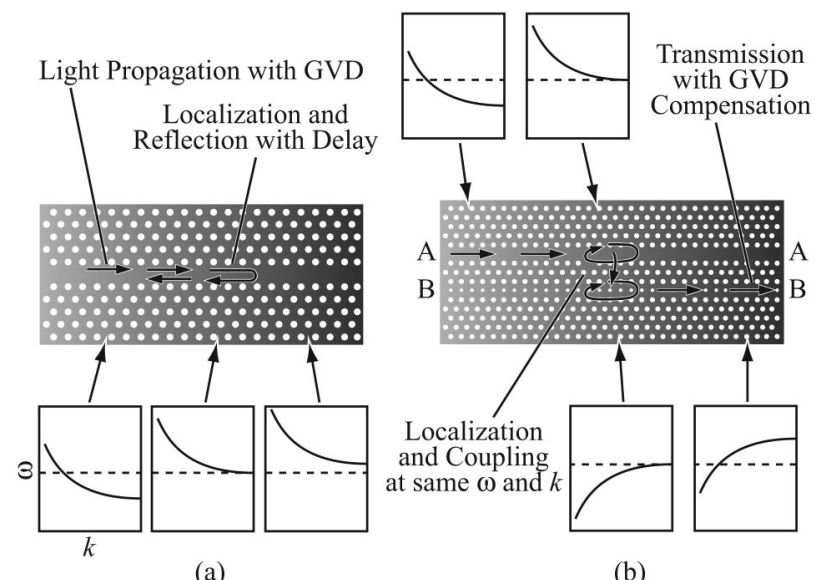

(a)

(b)

FIG. 1. Schematic diagrams of index-chirped PC waveguide devices. (a) Simple chirped waveguide. (b) Group delay device composed of two different chirped waveguides in a directional coupler structure. 


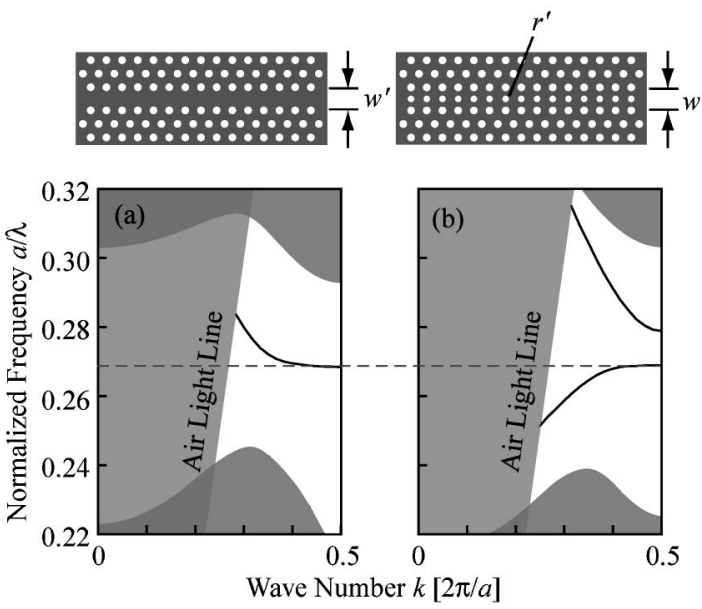

FIG. 2. Waveguide structures and photonic band diagrams calculated by the plane wave expansion method. In this calculation, $2 r / a=0.60$, channel width $w^{\prime}=0.85 w$, and the background index is fixed to 2.963. (a) Waveguide A. (b) Waveguide B with $2 r^{\prime} / a=0.505$.

edge is completely canceled. As a result, a transmission-type group delay device for an ultrashort pulse is realized. Not only zero GVD, but also any desired GVD, can also be given for the pulse by designing the band profiles to be asymmetric.

Figure 2 shows practical structures and photonic band diagrams of two waveguides with a background index of 2.963. Both waveguides have a typical normalized airhole diameter $2 r / a$ of 0.600 in the PC. Waveguide A has a channel of width $w^{\prime}$ of 0.85 times the normal width $w$ of single line missing airholes. In this waveguide, the band edge lies almost at the center of the PBG and an upward band of guided mode appears below the air light line. ${ }^{7}$ On the other hand, waveguide $\mathrm{B}$ has smaller airholes with normalized diameter $2 r^{\prime} / a$ at the center of the channel. In this waveguide, both upward and downward bands appear in the PBG below the air light line with a local gap. ${ }^{10}$ When $2 r^{\prime} / a=0.505$ and $w^{\prime}=0.85 w$ are assumed in waveguide $\mathrm{B}$, the band edges of the two waveguides agree, with an error of $\Delta(a / \lambda)<0.001$, and the band profiles are almost symmetric.

Two-dimensional FDTD calculation was carried out to confirm the device operation. The spatial distribution and input/output time wave forms of light intensity in the simple chirped waveguide are shown in Fig. 3(a). Here, $a$ $=0.380 \mu \mathrm{m}$ and $2 r=0.200 \mu \mathrm{m}(2 r / a=0.526)$. A Gaussian pulse was excited with a center wavelength $\lambda_{0}$ $=1.525 \mu \mathrm{m}\left(a / \lambda_{0}=0.249\right)$, a full width at half maximum (FWHM) $\Delta t=280 \mathrm{fs}$, and polarization inside the 2D plane. For this pulse width and a chirped length $L$ of $30 \mu \mathrm{m}$, the range of the index chirping was set to be from 2.963 to 2.850. As observed in the figure, the pulse gradually slows down, and its distribution is transformed so that the envelope corresponds to the Fourier spectrum of the pulse. Light localized by the low $v_{g}$ behaves as a standing wave but is soon reflected and begins to propagate backward in the waveguide with the inverse Fourier transformation of the distribution. Note that the initial pulse wave form is approximately maintained at the output end; $\Delta t$ of the reflected pulse is still 310 fs. A large GVD near the band edge is compensated by different path lengths for different frequencies, as in a chirped fiber grating. The optimum $\mathrm{L}$ for the dispersion compensation is uniquely determined from the band charac-

Downloaded 11 Aug 2004 to 134.76.85.86. Redistribution subject (a)

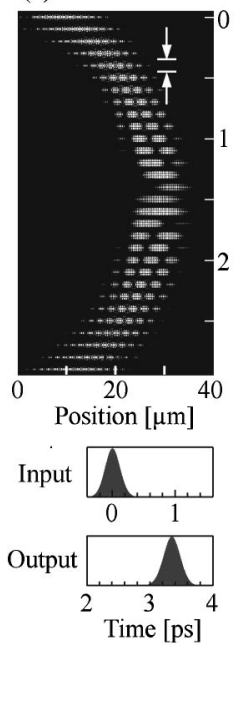

(b)

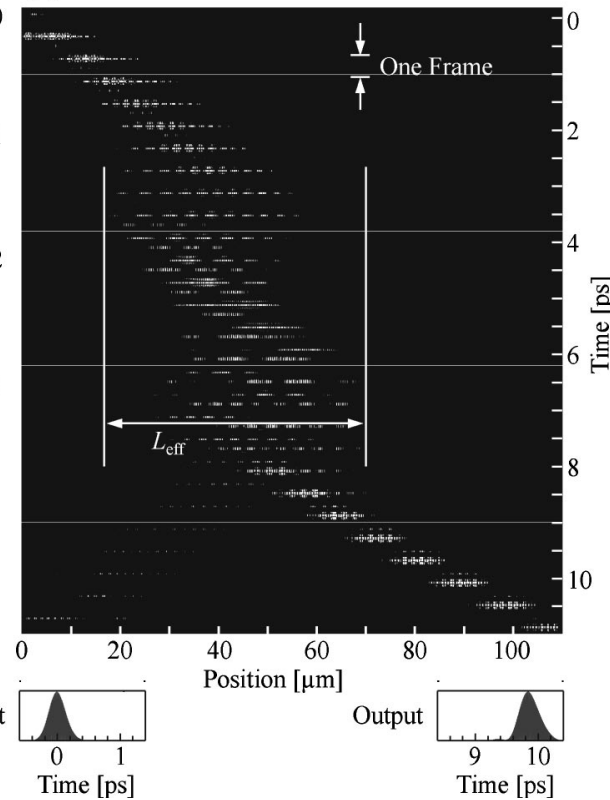

FIG. 3. Spatial distributions and input/output time wave forms of light intensity (square of the magnetic field normal to the 2D plane). An input pulse of $\Delta t=280 \mathrm{fs}$ is assumed. (a) Simple chirped waveguide with $L=30 \mu \mathrm{m}$. (b) Group delay device with $L=100 \mu \mathrm{m}$ and three airhole lines separating waveguides A and B. Nonchirped waveguides of $5 \mu \mathrm{m}$ length are added to both ends. The effective length $L_{\text {eff }}$ for light localization is measured to be $45 \mu \mathrm{m}$.

teristic. The localization is accompanied by a group delay time $\Delta T$, but $\Delta T$ is limited to $\sim 2$ ps due to the restriction of the length.

Figure 3(b) shows the results for the directional coupler of waveguides A and B. Here, $a=0.420 \mu \mathrm{m}, 2 r$ $=0.252 \mu \mathrm{m},(2 r / a=0.600), L=100 \mu \mathrm{m}$, and $\lambda_{0}=1.530 \mu \mathrm{m}$ $\left(a / \lambda_{0}=0.275\right)$. The index of the PC around waveguide $\mathrm{B}$ is slightly detuned by $<0.01$, since the precise matching of band edges is difficult due to the digitization error. Other parameters are the same as for Figs. 2 and 3(a). As observed in Fig. 3(b), the pulse propagating in waveguide A forms a standing wave with the envelope of the Fourier spectrum and then moves to waveguide B by the directional coupling. The pulse finally propagates forward in waveguide B with the inverse Fourier transformation. As expected from the above discussion, the GVD is well compensated. The weak reflection to the input waveguide and the slight expansion of the output pulse are caused by the small disagreement of the band edges and asymmetry of the two waveguide bands, which will be reduced by further fine control of band profiles. Since the assumed device includes an extra length for both input and output waveguides, the light localization occurs only near the center of the device within an effective length $L_{\text {eff }}$ of $53 \mu \mathrm{m}$ with a delay $\Delta T$ of $8.3 \mathrm{ps}$. The same calculation was carried out for various $L$ and $\Delta t$. Figure 4 summarizes calculated $\Delta T$ as functions of $L$ and $L_{\text {eff }}$ against $\Delta t=256 \mathrm{fs}, 1.09 \mathrm{ps}$, and $\infty$ (continuous wave). Note that $\Delta T$ increases almost linearly with $L$ and $L_{\text {eff }}$ This means that a longer delay will be possible by using a slowly chirped waveguide. For longer $\Delta t$, the same delay is obtained within a shorter $L_{\text {eff }}$ because of the narrower spectral width. The average $v_{g}$ in the effective length is estimated from the line slope to be $c / 56$ and $c / 100$ for $\Delta t=256$ fs and $109 \mathrm{ps}$, respectively. The slowest limit for a longer pulse is determined AlP license or copyright, see http://apl.aip.org/apl/copyright.jsp 


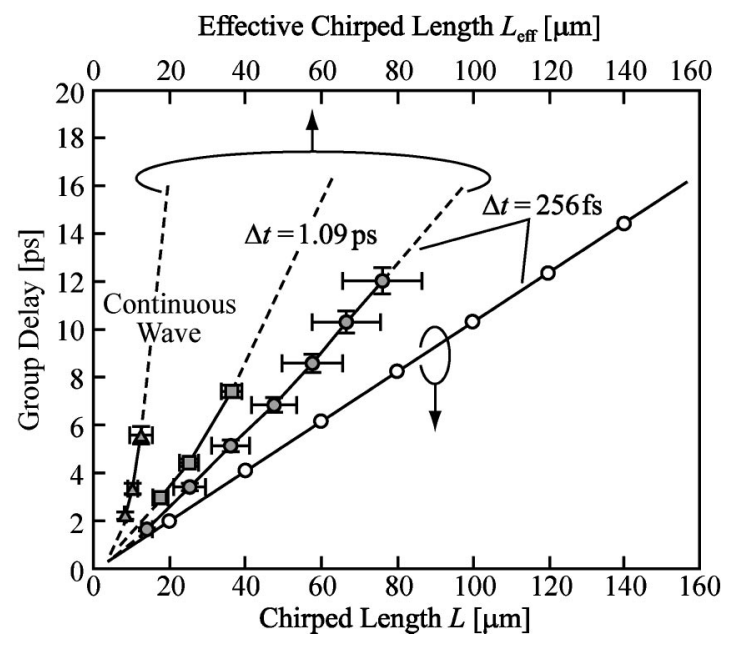

FIG. 4. Group delay time $\Delta T$ with chirped waveguide length $L$ and effective length $L_{\text {eff. }}$ The delay time is estimated from peak to peak of input and output pulse. Pulse width $\Delta t$ is taken as a parameter.

by the $v_{g}$ for the continuous wave, i.e., $<c / 350$. Therefore, a $1 \mathrm{~ns}$ delay will be possible for $L_{\mathrm{eff}}<1 \mathrm{~mm}$.

Finally, let us discuss how to realize the index chirping. The PC slab with tapered thickness is a candidate structure that passively forms the effective index chirping. For a material index of the slab of 3.5 and $\lambda=1.525 \mu \mathrm{m}$, effective indexes of 2.963-2.850 are obtained for thicknesses of $0.256-0.220 \mu \mathrm{m}$. Here, the precise control of the absolute thickness is not necessarily required, once the effective index of the taper covers all the spectrum of the input pulse. A more important issue for maintaining the pulse shape is the smoothness of the taper. A smooth taper will be possible by the tapered polishing and/or the nonuniform deposition of a high index film on top of the PC slab. However, the partial formation of arbitrary tapers will be a technical challenge. On the other hand, the active control will be realized by the gradient heating, gradient applied electric field, and so on. They are effective for realizing the smooth chirping. For example, a temperature change of $20^{\circ} \mathrm{C}$ gives an index change of $4 \times 10^{-3}$ in $\mathrm{Si}$. This value is much smaller than that as- sumed in the above simulation, but still sufficient for covering the spectral width of 3-10 ps pulse. If such active control makes the effective chirped length variable, the device acts as a tunable group delay device.

In conclusion, by using a directional coupler composed of two index-chirped PC waveguides having different band profiles, a unique group delay device can be realized. FDTD simulation suggested that a $1 \mathrm{~ns}$ group delay is possible within an effective device length of $<1 \mathrm{~mm}$. A significant advantage of this device is that it works even for a short pulse having a wide spectrum without changing its wave form. This property is essentially different from simple low $v_{g}$ light propagation at the band edge. If the external control of the chirping is realized, a tunable device will be possible. This device is also expected to provide various other applications, such as optical buffer memories, efficient optical switches and nonlinear devices.

This study was supported by the IT Program and the 21st COE Program, both through the Ministry of Education, Culture, Sports, Science and Technology. Additional support was provided by CREST of the Japan Science and Technology Corporation.

${ }^{1}$ T. Baba, N. Fukaya, and J. Yonekura, Electron. Lett. 35, 654 (1999); T. Baba, A. Motegi, T. Iwai, N. Fukaya, Y. Watanabe and A. Sakai, IEEE J. Quantum Electron. 38, 743 (2002).

${ }^{2}$ M. Lončar, D. Nedeljković, T. Doll, J. Vučković, A. Scherer, and T. P. Pearsall, Appl. Phys. Lett. 87, 952 (2000).

${ }^{3}$ C. J. Smith, H. Benisty, S. Olivier, M. Rattier, C. Weisbuch, T. F. Krauss, R. M. De La Rue, R. Houdré, and U. Oesterle, Appl. Phys. Lett. 77, 2813 (2000).

${ }^{4}$ M. Notomi, A. Shinya, K. Yamada, J. Takahashi, C. Takahashi, and I. Yokohama, Electron. Lett. 37, 293 (2001).

${ }^{5}$ A. Mekis, J. C. Chen, I. Kurland, S. Fan, P. R. Villeneuve, and J. D. Joannopoulos, Phys. Rev. Lett. 77, 3787 (1996).

${ }^{6}$ J. Yonekura, M. Ikeda, and T. Baba, J. Lightwave Technol. 17, 1500 (1999).

${ }^{7}$ M. Notomi, K. Yamada, A. Shinya, J. Takahashi, C. Takahashi, and I. Yokohama, Phys. Rev. Lett. 87, 253902 (2001).

${ }^{8}$ T. Baba, D. Mori, K. Inoshita, and Y. Kuroki, IEEE J. Sel. Top. Quantum Electron. (to be published).

${ }^{9}$ M. F. Yanik and S. Fan, Phys. Rev. Lett. 92, 083901 (2004).

${ }^{10}$ K. Yamada, M. Notomi, A. Shinya, C. Takahashi, and H. Morita, Electron. Lett. 38, 74 (2002). 